\title{
Hausse des cas de méningococcie invasive Neisseria meningitidis ST-11 de sérogroupe W au Canada, de 2016 à 2018
}

\author{
RS Tsang 1*, L Hoang2 ${ }^{2}$ GJ Tyrrell33, J Minion4, P Van Caeseele ${ }^{5}$, JV Kus ${ }^{6,7}$, B Lefebvre, D Haldane ${ }^{9,10}$, \\ R Garceau ${ }^{11}$, G German ${ }^{12}$, G Zahariadis ${ }^{13,14}$, B Hanley ${ }^{15}$
}

\section{Résumé}

Contexte : De nombreux pays ont enregistré une hausse des cas de méningococcie invasive causée par une souche de Neisseria meningitidis de sérogroupe W (MenW) du complexe clonal (CC) de séquence type multilocus (ST)11. Le premier cas canadien de MenW a été signalé en Ontario en 2014. En 2016, cette souche avait entraîné des cas de méningococcie invasive dans cinq provinces et était responsable de $18,8 \%$ des cas de méningococcie invasive au Canada.

Objectif : Faire le bilan sur l'infection à MenW invasive au Canada, notamment les caractéristiques de cette souche, la source des isolats du spécimen, l'âge, le sexe et la répartition géographique des cas.

Méthodologie : Des $N$. meningitidis provenant de cas de méningococcie invasive à culture positive sont régulièrement envoyées au Laboratoire national de microbiologie (LNM) aux fins d'analyse de sérogroupe, sérotype, séro-sous-type et type de séquence. Les données du

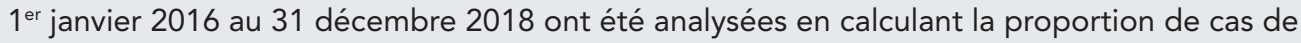
méningococcie invasive causées par la MenW par rapport aux autres sérogroupes. De plus, des tendances fondées sur l'âge, le sexe et la répartition géographique des cas et de la source des isolats ont été analysées en fonction des renseignements figurant sur les formulaires de demande de spécimen.

Résultats : Au cours de cette période de trois ans, 292 isolats individuels de méningococcie invasive ont été analysés. Le pourcentage d'isolats de méningococcie invasive de type MenW a plus que doublé, passant de $19 \%(n=15)$ à $44 \%(n=51)$ en 2018 , lorsque la MenW est devenue le sérogroupe le plus courant, dépassant le nombre de souches MenB, MenC et MenY. Au total, 93 isolats de MenW ont été relevés; $91 \%(n=85)$ appartenant au CC ST-11. Cette hausse de la MenW a touché toutes les tranches d'âge (mais était plus courante chez les personnes âgées de plus de 60 ans), les deux sexes et a été enregistrée dans l'ensemble du pays, mais de manière plus fréquente dans l'ouest du Canada. La source de spécimen la plus courante était le sang.

Conclusion : En 2018, MenW était le sérogroupe le plus courant pour les isolats reçus au LNM provenant de cas de méningococcie invasive à culture positive au Canada. Plus de $90 \%$ des isolats du sérogroupe MenW appartenaient au CC ST-11. Le vaccin anti-méningococcique conjugué quadrivalent $A C W Y$ protège contre la méningococcie invasive causée par des souches des sérogroupes $A, C, W$ et $Y$ et peut donc protéger contre la méningococcie invasive causée par la nouvelle souche MenW ST-11; de plus amples recherches sont cependant nécessaires. L'émergence d'autres souches souligne l'importance de la caractérisation de la souche en matière de surveillance et de recherches relatives à la méningococcie invasive.
Cette oeuvre est mise à la disposition selon les termes de la licence internationale Creative Commons Attribution 4.0

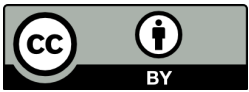

Affiliations

${ }^{1}$ Laboratoire national de microbiologie, Agence de la santé publique du Canada, Winnipeg (Manitoba)

2 BC Public Health Microbiology and Reference Laboratory, Vancouver (Colombie-Britannique)

${ }^{3}$ Provincial Laboratory for Public Health, Edmonton (Alberta)

${ }^{4}$ Saskatchewan Disease Control Laboratory, Regina (Saskatchewan)

${ }^{5}$ Laboratoire provincial Cadham, Winnipeg (Manitoba)

${ }^{6}$ Santé publique Ontario, Toronto (Ontario)

${ }^{7}$ Department of Laboratory Medicine and Pathobiology, Université de Toronto, Toronto (Ontario)

${ }^{8}$ Laboratoire de santé publique du Québec, Institut national de santé publique du Québec, Sainte-Anne-de-Bellevue (Québec)

${ }^{9}$ Régie de la santé de la Nouvelle-Écosse, Halifax (Nouvelle-Écosse)

${ }^{10}$ Université Dalhousie, Halifax (Nouvelle-Écosse)

${ }^{11}$ Unité de contrôle des maladies transmissibles, Ministère de la Santé, gouvernement du Nouveau-Brunswick, Fredericton (Nouveau-Brunswick)

${ }^{12}$ Ministère de la Santé, gouvernement de l'île-du-Prince-Édouard,

Charlottetown (Île-du-Prince-Édouard)

${ }^{13}$ Provincial Public Health Laboratory, Eastern Health Microbiology Services, St. John's (Terre-Neuve-et-Labrador)

${ }^{14}$ Department of Laboratory Medicine, Faculty of Medicine, Université Memorial de Terre-Neuve, St. John's (Terre-Neuve-et-Labrador)

${ }^{15}$ Centre de lutte contre les maladies transmissibles, Ministère de la santé et des affaires sociales,

Whitehorse (Yukon)

*Correspondance :

raymond.tsang@canada.ca 
Citation proposée : Tsang RS, Hoang L, Tyrrell GJ, Minion J, Van Caeseele P, Kus JV, Lefebvre B, Haldane D,

Garceau R, German G, Zahariadis G, Hanley B. Hausse des cas de méningococcie invasive Neisseria

meningitidis ST-11 du sérogroupe W au Canada, de 2016 à 2018. Relevé des maladies transmissibles au

Canada 2019;45(6):181-7. https://doi.org/10.14745/ccdr.v45i06a04f

Mots-clés : méningococcie invasive, Neisseria meningitidis, type de séquence, complexe clonal, type de

séquence 11 , sérogroupe W, Canada

\section{Introduction}

La méningococcie invasive infection invasive à méningocoque causée par la bactérie Neisseria meningitidis est une maladie à déclaration obligatoire (MADO) dans la plupart des pays, notamment le Canada, ainsi que selon l'Organisation mondiale de la Santé (1). Les formes les plus graves de la méningococcie invasive comprennent la méningite et la septicémie, mais cette bactérie peut également entraîner une pneumonie, de l'arthrite septique et des infections localisées comme une conjonctivite et l'urétrite. Avant l'introduction de la vaccination courante contre le sérogroupe méningococcique $\mathrm{C}$ (MenC) au Canada, les taux d'incidence de méningococcie invasive étaient d'environ 1,0 cas pour 100000 habitants; les taux augmentant lors d'éclosions d'infections à MenC (2). Entre 1989 et 1993, par exemple, l'incidence de méningococcie invasive étaient de 1,4 à 1,6 cas pour 100000 habitants (3). Depuis I'introduction des vaccins conjugués contre la MenC au milieu des années 2000, le taux d'incidence moyen global de méningococcie invasive au Canada a diminué pour s'établir à environ 0,6 pour 100000 habitants, la plupart des cas étant dus aux souches MenB et MenY. Historiquement, la MenW n'est pas une cause majeure de méningococcie invasive (4).

Au Canada, la plupart des cas de méningococcie invasive sont confirmés par culture et/ou réaction en chaîne de la polymérase (PCR) dans des hôpitaux locaux et des laboratoires provinciaux de la santé publique provinciaux. Des isolats de la $N$. meningitidis invasive sont également couramment soumis au Laboratoire national de microbiologie (LNM) pour typage plus approfondit, notamment aux fins d'analyse antigénique et d'analyse clonale génétique.

\section{Classification de la bactérie Neisseria meningitidis}

Douze sérogroupes $N$. meningitidis ont été relevés en fonction de l'antigénicité de leurs polysaccharides capsulaires de surface. La plupart des méningococcie invasives sont causées par les six sérogroupes $A(M e n A), B(M e n B), C(M e n C), W(M e n W)$, $X(M e n X)$ et $Y(M e n Y)(5,6)$. La prévalence des divers sérogroupes varie en fonction des régions géographiques (5) et parfois également selon les programmes de vaccination locaux (7).

Outre le polysaccharide capsulaire du sérogroupe contribuant à la virulence, la plupart des souches pouvant causer des éclosions ou des épidémies de méningococcie invasive appartiennent à un nombre limité de clones ou de lignées hypervirulents et sont classées par séquence type (ST). Les types de séquence apparentés sont regroupés en complexes clonaux (CC). Certains des clones hypervirulents bien établis ont été décrits sous les formes ST-11, ST-32 et autres types de séquence $(5,8)$. Deux antigènes protéiques de la membrane externe, PorB et PorA, servent à classer plus précisément les souches, respectivement en sérotypes et séro-sous-types (9). Lorsque ces protéines de membrane externe sont utilisées avec l'antigène capsulaire, les souches peuvent être classées selon leur formule antigénique, par exemple, sérogroupe $B$ : sérotype 4 : séro-sous-type $P 1.7,4$. Cela permet d'effectuer le suivi de multiples variantes ainsi que l'épidémiologie de cette maladie.

\section{Émergence de la méningite MenW}

Des données recueillies au LNM au cours des cinq dernières années ont signalé l'émergence d'une souche clone ST-11 MenW au Canada, se caractérisant par les antigènes W:2a:P1.5,2. Ce clone a été détecté pour la première fois en Ontario en 2014 (10). En 2016, quatre autres provinces avaient déclaré des cas de méningococcie invasive due à ce clone MenW (11).

Dans cette étude, nous présentons la caractérisation des isolats de cette méningococcie invasive soumis au LNM entre 2016 et 2018. Nous avons analysé les souches par typage antigénique et génétique ainsi que les tendances en fonction de l'âge, du sexe, de la répartition géographique des cas et de la source des spécimens.

\section{Méthodologie Isolats bactériens}

Des N. meningitidis invasives cultivées et identifiées dans des hôpitaux locaux et des laboratoires de diagnostic sont couramment soumises à leur laboratoire de santé publique provincial respectif aux fins de détermination ou de confirmation du sérogroupe. Les provinces et les territoires transmettent de tels isolats au LNM pour un typage supplémentaire dans le cadre de la surveillance nationale de la méningococcie invasive.

La présente étude a utilisé des isolats de $N$. meningitidis obtenus de cas individuels de méningococcie invasive et n'a pas inclus de cas diagnostiqués par PCR ou détection d'antigène. La plupart des isolats que reçoit le LNM proviennent de cas de méningococcie invasive à culture positive. Des études antérieures ont indiqué que $90 \%$ des cas des de méningococcie invasive relevés par les provinces et territoires correspondaient à des isolats reçus au LNM $(12,13)$, ce qui suggère que le LNM possède un échantillon robuste et représentatif. 
La présente étude a inclus tous les isolats $N$. meningitidis invasifs obtenus de cas de méningococcie invasive confirmée par culture soumis au LNM entre le $1^{\text {er }}$ janvier 2016 et le 31 décembre 2018. Les isolats en double de mêmes patients ont été retirés de l'analyse.

\section{Typage de la Neisseria meningitidis}

Au LNM, les isolats ont été caractérisés par des méthodes standards, afin de déterminer leur formule antigénique (sérogroupe:sérotype:séro-sous-type). Le typage par séquençage multilocus (MLST) a été effectué selon les procédures précédemment décrites $(14,15)$ et les isolats ont été classés en types de séquence et complexes clonaux conformément aux procédures décrites sur le site Web Neisseria MLST (16).

\section{Données démographiques et source de spécimen}

Les renseignements sur l'âge et le sexe de chaque patient ainsi que l'origine géographique et la source de chaque spécimen proviennent de formulaires de demande fournis avec les spécimens par les laboratoires de santé provinciaux publics. Pour l'origine géographique, nous avons combiné les données pour le Canada atlantique (Nouveau-Brunswick, Terre-Neuve-et-Labrador, Nouvelle-Écosse, île-du-Prince-Édouard) ainsi que pour le Nord du Canada (Territoires du Nord-Ouest, Nunavut, Yukon).

\section{Résultats}

Entre le $1^{\text {er }}$ janvier 2016 et le 31 décembre 2018, 292 isolats de cas individuels au total ont été envoyés au LNM : 80 en 2016, 97 en 2017 et 115 en 2018.

\section{Répartition des sérogroupes}

La répartition des sérogroupes des cas de méningococcie invasive confirmée par culture a varié au cours de cette période de trois ans. Le nombre d'isolats de MenC est demeuré faible, fluctuant entre trois et huit isolats par an. La proportion des isolats de méningococcie invasive de type MenB et MenY a enregistré une tendance à la baisse au cours de cette période de trois ans. Le nombre d'isolats de type MenW a augmenté, passant de 15 en 2016 à 51 en 2018 et a dépassé le nombre d'isolats MenB ou MenY en 2018 (figure 1)

\section{Caractéristiques des souches}

Sur les 93 isolats de MenW, 85 (91\%) ont été déterminés comme de CC ST-11, six (6 \%) de CC ST-22 et un de CC ST-60 (ST-11739); un isolat n'a été affecté à aucun complexe clonal connu (ST-1308). Le type ST-11 a été déterminé pour la majorité ( $n=83$, soit $98 \%$ ) des isolats de MenW CC ST-11; seulement deux ont été identifiés comme variantes à locus unique de ST-11. Parmi ces variantes, l'une était de type ST-12818 et l'autre de type ST-13250. II a été déterminé que la plupart des MenW de CC T-11 présentaient l'antigène de sérotype $2 a(n=76 ; 89 \%)$
Figure 1 : Répartition des sérogroupes de Neisseria meningitidis provenant de cas d'infections invasives à méningocoque au Canada, de 2016 à 2018

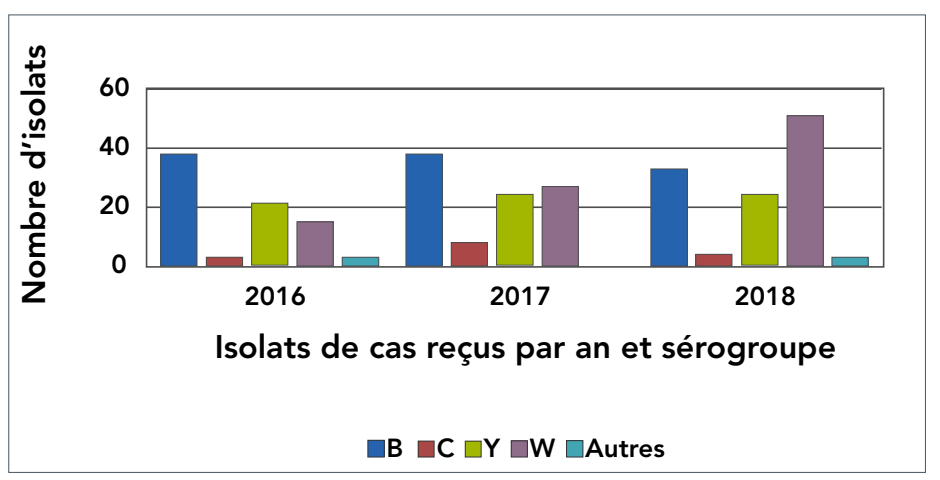

et la plupart était de type 2a:P1.5,2 ( $n=42 ; 49 \%$ ) ou 2a:P1.2 ( $n=31 ; 36 \%$ ). Les six MenW de CC ST-22 étaient de type NT (non typable):P1.6. La MenW ne pouvant être classée dans aucun des CC connus a reçu le type 4:P1.16 et la MenW de CC ST-60 a été typée $2 \mathrm{a}: \mathrm{P1}$.5,2.

Cinq types de séquence ont été relevés parmi les six MenW de CC ST-22 : un isolat de ST-22, deux de ST-184 et un de ST-1158, de ST-1124 et de ST-8974. Pour les cinq isolats non ST-22, six des sept gènes constitutifs des types de séquence étaient identiques à ceux de ST-22 (variantes à locus unique de ST-22).

\section{Données démographiques et source de spécimen}

Entre 2016 et 2018, plus de la moitié ( $n=55,65 \%$ ) des cas de MenW CC ST-11 concernait des adultes de plus de 30 ans (notamment 34 cas, cest-à-dire $40 \%$, de plus de 60 ans), alors que $19 \%(n=16)$ étaient de 16 à 30 ans (tableau 1$)$.

Tableau 1 : Répartition selon l'âge et le sexe des de méningococcie invasive causée par le sérogroupe $\mathbf{W}$ Neisseria meningitidis (MenW) de complexe clonal ST-11, de 2016 à 2018, au Canada

\begin{tabular}{|c|c|c|c|c|c|}
\hline \multirow{2}{*}{\multicolumn{2}{|c|}{$\begin{array}{c}\text { Âge et sexe des } \\
\text { cas }\end{array}$}} & \multicolumn{3}{|c|}{ Nombre de cas par an } & \multirow{2}{*}{$\begin{array}{l}\text { Nombre } \\
\text { total de } \\
\text { cas }\end{array}$} \\
\hline & & 2016 & 2017 & 2018 & \\
\hline \multirow{2}{*}{ Sexe } & Homme & 9 & 12 & 18 & 39 \\
\hline & Femme & 4 & 13 & 29 & 46 \\
\hline \multirow{7}{*}{ Âge } & $\begin{array}{l}\text { Moins de } \\
12 \text { mois }\end{array}$ & 0 & 1 & 5 & 6 \\
\hline & 1 à 5 ans & 1 & 0 & 4 & 5 \\
\hline & 6 à 15 ans & 1 & 2 & 0 & 3 \\
\hline & 16 à 30 ans & 2 & 7 & 7 & 16 \\
\hline & 31 à 60 ans & 3 & 5 & 13 & 21 \\
\hline & $\begin{array}{l}\text { Plus de } 60 \\
\text { ans }\end{array}$ & 6 & 10 & 18 & 34 \\
\hline & $\begin{array}{l}\text { Tous les } \\
\text { âges }\end{array}$ & 13 & 25 & 47 & 85 \\
\hline
\end{tabular}


En 2018, neuf (19\%) des 47 cas étaient de moins de six ans; cinq étant de moins de 12 mois (tableau 1). En revanche, seuls deux cas de moins de six ans ont été enregistrés pour la période combinée de 2016 et 2017. Sur les 85 cas au cours de la période de l'étude, 46 étaient des femmes.

L'augmentation de la MenW a été particulièrement significative dans I'Ouest du Canada (tableau 2). La proportion de MenW parmi tous les isolats de méningococcie invasive entre 2016 et 2018 était de $57 \%$ en Colombie-Britannique, 50 \% en Alberta, $43 \%$ en Saskatchewan et $46 \%$ au Manitoba, alors que les pourcentages globaux des isolats de méningococcie invasive de type MenW étaient de $25 \%$ en Ontario et $17 \%$ au Québec. Dans le Canada atlantique, seul un isolat de cas de MenW a été enregistré au cours de cette même période de trois ans. Le LNM n'a reçu qu'un seul MenW sur un total de deux isolats de méningococcie invasive du Nord du Canada.

La source des spécimens des MenW de CC ST-11 a été principalement des hémocultures ( $n=79$ ou $93 \%$ ); trois ont été trouvés dans du liquide céphalorachidien (y compris un à la fois dans du sang et du liquide céphalorachidien). En 2018, deux cas de CC MenW ST-11 ont été trouvés dans du liquide synovial; pour un cas, l'organisme a été isolé d'un spécimen sous-arachnoïdien.

\section{Discussion}

Au cours des trois dernières années, le nombre d'isolats de N. meningitidis invasive reçus au LNM a augmenté de $44 \%$, passant de 80 isolats en 2016 à 115 en 2018. Cette hausse est principalement due à la bactérie MenW, dont le nombre d'isolats a plus que triplé, passant de 15 en 2016 à 51 en 2018. Cette augmentation de la MenW a modifié la répartition des sérogroupes de méningococcie invasive au Canada, qui, depuis I'introduction des vaccins conjugués contre la MenC, était principalement la MenB. La hausse de la MenW au Canada est due à l'expansion et à la diffusion continues de I'hypervirulent CC ST-11. Cette augmentation a été particulièrement évidente dans les provinces de l'Ouest, où la MenW a représenté de $43 \%$ à $57 \%$ des isolats de méningococcie invasive.

Ces isolats de MenW de CC ST-11 au Canada sont similaires aux souches circulant actuellement dans le monde (17). Des découvertes parallèles ont été signalées en Australie (18), dans des régions des États-Unis $(19,20)$ et en République centrafricaine (21). En Australie, $44 \%$ des cas de méningococcie invasive en 2016 étaient dus à la MenW; c'est-à-dire de près du triple depuis 2015 (18). En République centrafricaine, 65 \% des cas de méningococcie invasive à culture positive ont été dus à la

Tableau 2 : Répartition temporelle et géographique des méningococcies invasive de culture positive pour des isolats de tous les sérogroupes et du sérogroupe W Neisseria meningitidis (MenW) au Canada, selon le complexe clonal $^{a}$

\begin{tabular}{|c|c|c|c|c|c|c|c|c|c|c|}
\hline \multirow[b]{2}{*}{ Région } & \multicolumn{3}{|c|}{2016} & \multicolumn{3}{|c|}{2017} & \multicolumn{3}{|c|}{2018} & 2016 à 2018 \\
\hline & $\begin{array}{l}\text { Tous les } \\
\text { isolats } \\
\text { de MI }\end{array}$ & $\begin{array}{c}\text { MenW } \\
\text { ST-11 }\end{array}$ & $\begin{array}{c}\text { MenW } \\
\text { non } \\
\text { ST-11 }\end{array}$ & $\begin{array}{l}\text { Tous les } \\
\text { isolats } \\
\text { de MI }\end{array}$ & $\begin{array}{l}\text { MenW } \\
\text { ST-11 }\end{array}$ & $\begin{array}{c}\text { MenW } \\
\text { non ST-11 }\end{array}$ & $\begin{array}{l}\text { Tous les } \\
\text { isolats } \\
\text { de MI }\end{array}$ & $\begin{array}{l}\text { MenW } \\
\text { ST-11 }\end{array}$ & $\begin{array}{l}\text { MenW non } \\
\text { ST-11 }\end{array}$ & $\begin{array}{c}\text { Nombre total } \\
\text { d'isolats } \\
\text { de Ml }\end{array}$ \\
\hline
\end{tabular}

\begin{tabular}{|c|c|c|c|c|c|c|c|c|c|c|}
\hline C.-B. & 11 & 2 & 0 & 23 & 13 & 1 & 22 & 14 & 2 & 56 \\
\hline Alb. & 7 & 1 & 1 & 8 & 4 & 0 & 19 & 11 & 0 & 34 \\
\hline $\begin{array}{l}\text { Man., } \\
\text { Sask. }\end{array}$ & 6 & 2 & 1 & 8 & 2 & 0 & 6 & 3 & 1 & 20 \\
\hline Ont. & 24 & 5 & 0 & 26 & 4 & 0 & 31 & 11 & 0 & 81 \\
\hline Qc & 25 & 3 & 0 & 24 & 2 & 1 & 26 & 6 & 1 & 75 \\
\hline $\begin{array}{l}\text { N.-B., } \\
\text { T.-N. L., } \\
\text { N.-É.' } \\
\text { I.-P.-É. }\end{array}$ & 6 & 0 & 0 & 8 & 0 & 0 & 10 & 1 & 0 & 24 \\
\hline $\begin{array}{l}\text { T.N.-O., } \\
\text { Nt, Yn }\end{array}$ & 1 & 0 & 0 & 0 & 0 & 0 & 1 & 1 & 0 & 2 \\
\hline Total & 80 & 13 & 2 & 97 & 25 & 2 & 115 & 47 & 4 & 292 \\
\hline
\end{tabular}

Abréviations : Alb., Alberta; C.-B., Colombie-Britannique; Man., Manitoba; MenW, Neisseria meningitidis de sérogroupe W; MI, méningococcie invasive; N.-B., Nouveau-Brunswick;

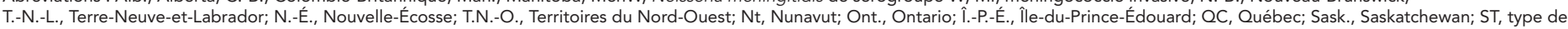
T.-N.-L., Terre-Neuve-et-Labrador;
séquence; $Y n$, territoire du Yukon

a Soit le complexe clonal ST-11 (ST-11) ou le complexe clonal non ST-11 (non ST-11) 
MenW; l'âge médian des patients touchés par la MenW étant de 60 ans en 2015 et de 66 ans en 2016 (21).

La prédominance de la souche CC ST-11 est relativement nouvelle, mais des changements émergent dans son antigène de sérotype. Avant 2014, la souche prédominante était le CC ST-22 (10,11). Entre 2009 et 2016, 100 \% des MenW de CC ST-11 présentaient l'antigène de sérotype 2a. Entre 2017 et 2018, cependant, neuf des 72 isolats (13\%) n'exprimaient pas I'antigène de sérotype $2 a$. Auparavant, nous avions observé un point chaud de mutation de l'antigène de sérotype $2 \mathrm{a}$ des souches MenC (22).

Notre découverte d'un CC ST-60 invasif (isolat W:2a:P1.5,2 de ST-11739) dans la MenW était inhabituelle, puisque la plupart des méningococcies de CC ST-60 au Canada sont dues à la MenB $(23,24)$ et les études antérieures de la MenC ou de la MenW exprimant l'antigène de sérotype 2a étaient principalement de type CC ST-11 $(11,25)$. D'autres études sont nécessaires pour comprendre l'origine de cette souche W:2a:P1.5,2 ST-11739 inhabituelle.

\section{Limites}

Certaines limites de notre étude doivent être prises en compte lors de l'interprétation des résultats. Tout d'abord, nous avons uniquement examiné des cas confirmés par culture et n'avons pas inclus de cas diagnostiqués par des moyens moléculaires uniquement (c.-à-d. PCR). Même si cela peut influer sur la proportion des cas de méningococcie invasive causés par la MenW, les cas diagnostiqués par PCR ne représentant qu'environ $10 \%$ de tous les cas de méningococcie invasive relevés au Canada (4), l'effet général sur les résultats ne devrait probablement pas modifier la tendance actuelle de l'augmentation des cas de méningococcie invasive MenW.

Ensuite, nous n'avons pas recueilli de données cliniques ni relevé l'évolution de la maladie pour les cas de MenW et ne savons donc pas si les taux de mortalité étaient aussi élevés que ceux déclarés dans d'autres pays ou régions ( $24 \%$ en Géorgie aux États-Unis, 20 \% en Angleterre au Royaume-Uni et $21 \%$ en Australie) $(19,26,27)$. De la même manière, nous ne savons pas si les cas canadiens présentaient un tableau clinique inhabituel, comme des symptômes gastro-intestinaux ou une épiglottite $(26,28)$.

\section{Répercussions cliniques et de santé publique}

Le vaccin anti-méningococcique conjugué quadrivalent ACWY protège contre la méningococcie invasive causée par des souches exprimant les antigènes des sérogroupes $A, C, W$ ou Y. En théorie, il devrait donc protéger contre la méningococcie invasive causée par la souche MenW ST-11. En 2017, une épidémie en Colombie-Britannique a incité à l'immunisation des personnes âgées de 15 à 19 ans dans la région touchée avec le vaccin anti-méningococcique conjugué quadrivalent ACWY (29). Cette immunisation a eu lieu en plus du rappel courant du vaccin anti-méningococcique conjugué quadrivalent administré aux élèves de neuvième année, mis en œuvre en septembre 2016 (30). Bien que la plupart des provinces et des territoires disposent désormais de programmes de vaccin anti-méningococcique conjugué quadrivalent $A C W Y$ pour les élèves d'établissements primaires ou secondaires (31), certaines populations ne sont pas couvertes, notamment les adultes âgés de plus de 30 ans, la tranche d'âge la plus touchée au cours des trois dernières années de la collecte de données.

\section{Conclusion}

Entre 2016 et 2018, on a observé une hausse des cas de MenW invasive qui a modifié la répartition des sérogroupes de cas de méningococcie invasive confirmés par culture au Canada. Le vaccin anti-méningococcique conjugué quadrivalent ACWY protège contre la méningococcie invasive causée par des souches des sérogroupes $A, C, W$ ou $Y$. Ce vaccin peut également protéger contre la méningococcie invasive causée par la nouvelle souche MenW ST-11, mais des recherches supplémentaires sont nécessaires. L'émergence de types clonaux plus récents et de souches inhabituelles souligne l'importance de la caractérisation de la souche en matière de surveillance et de recherches relatives à la méningococcie invasive.

\section{Déclaration des auteurs}

Tous les auteurs (R. S. T., L. H., G. J. T., J. M., P. V. C., J. V. ., B. L., D. H., R. G., G. G., G. Z. et B. H.) participent à la surveillance de la méningococcie invasive au Canada. R. S. T. a préparé la première version et tous les auteurs ont contribué à la version finale au moyen de commentaires et de suggestions.

\section{Conflit d'intérêts}

Aucun.

\section{Remerciements}

Nous remercions le personnel des laboratoires provinciaux de santé publique d'avoir identifié et soumis des isolats de N. meningitidis au Laboratoire national de microbiologie. Nous remercions en outre D. Law, J. Zhou et S. Deng d'avoir fourni une assistance laboratoire pour l'analyse des souches ainsi que le service essentiel d'ADN du LNM d'avoir fourni de l'aide pour la détermination des séquences nucléotidiques.

Pour la présente publication, nous avons eu recours au site Web de typage de séquence multilocus de Neisseria (16) développé par K. Jolley et hébergé par l'université d'Oxford. Le développement de ce site a été financé par le Wellcome Trust et I'Union européenne. 


\section{Aide financière}

La surveillance par laboratoire de la méningococcie invasive est soutenue par l'Agence de la santé publique du Canada.

\section{Références}

1. Anker M, Schaaf D. WHO report on global surveillance of epidemic-prone infectious diseases. Geneva: World Health Organization; 2000.

2. Pollard AJ, Tam TW. Une déclaration d'un comité consultative (DCC), Comité consultative national de I'immunisation (CCNI). Déclaration sur l'utilisation recommandée des vaccins antiméningococciques. Relevé des maladies transmissibles au Canada 2001;27(ACS-6):2-36. http://publications.gc.ca/collections/Collection/H12-21-2-275-6.pdf

3. Squires SG, Deeks SL, Tsang RS. Surveillance accrue des méningococcies invasives au Canada : du $1^{\text {er janvier } 1999}$ au 31 décembre 2001. Relevé des maladies transmissibles au Canada 2004 fév;30(3):17-28. https://www.canada.ca/fr/ sante-publique/services/rapports-publications/releve-maladie s-transmissibles-canada-rmtc/numero-mensuel/2004-30/ table-matieres-rmtc-30-03.html

4. Li YA, Tsang R, Desai S, Deehan H. Surveillance accrue des méningococcies invasives au Canada, 2006-2011. Relevé des maladies transmissibles au Canada 2014 May;40(9):184-94. $\mathrm{DOI}$

5. Harrison LH, Trotter CL, Ramsay ME. Global epidemiology of meningococcal disease. Vaccine 2009 Jun;27 Suppl 2:B5163. DOl PubMed

6. Xie O, Pollard AJ, Mueller JE, Norheim G. Emergence of serogroup $X$ meningococcal disease in Africa: need for a vaccine. Vaccine 2013 Jun;31(27):2852-61. DOI PubMed

7. Stefanelli P, Rezza G. Impact of vaccination on meningococcal epidemiology. Hum Vaccin Immunother 2016 Apr;12(4):1051-5. DOI PubMed

8. Caugant DA. Population genetics and molecular epidemiology of Neisseria meningitidis. APMIS 1998 May;106(5):505-25. DOl PubMed

9. Frasch CE, Zollinger WD, Poolman JT. Serotype antigens of Neisseria meningitidis and a proposed scheme for designation of serotypes. Rev Infect Dis 1985 Jul-Aug;7(4):504-10. DOl PubMed
10. Tsang RSW, Deeks SL, Wong K, Marchand-Austin A, Jamieson FB. I. L'infection invasive à Neisseria meningitidis de sérogroupe W (MenW) en Ontario (Canada) montre un remplacement clonal potentiel pendant la période

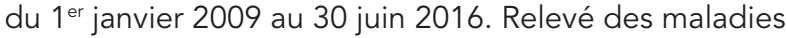
transmissibles au Canada 2016; 42(12):292-6. DOI

11. Tsang R, Hoang L, Tyrrell GJ, Horsman G, Van Caeseele $P_{1}$ Jamieson F, Lefebvre B, Haldane D, Gad RR, German GJ, Zahariadis $\mathrm{G}$. Augmentation des infections invasives à Neisseria meningitides de sérogroupe W au Canada de 2009 à 2016. Relevé des maladies transmissibles au Canada 2017 Jul;43(7/8):161-7. DOl

12. Navarro C, Deeks SL, Medaglia A, Tsang RS. Surveillance accrue des méningococcies invasives au Canada, du

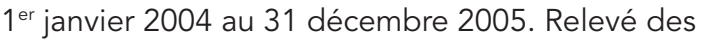
maladies transmissibles au Canada 2007 Jun;33(10):1-15. https://www.canada.ca/fr/sante-publique/services/ rapports-publications/releve-maladies-transmissibles-canadarmtc/numero-mensuel/2007-33/surveillance-accru e-meningococcies-invasives-canada.html

13. Watkins KM, Deeks SL, Medaglia A, Tsang RS. Surveillance accrue des méningococcies invasives au Canada, du $1^{\text {er }}$ janvier 2002 au 31 décembre 2003. Relevé des maladies transmissibles au Canada 2006 Apr;32(8):97-107.

https://www.canada.ca/fr/sante-publique/services/ rapports-publications/releve-maladies-transmissibles-canadarmtc/numero-mensuel/2006-32/table-matieres-rmtc-32-08. html

14. Maiden MC, Bygraves JA, Feil E, Morelli G, Russell JE, Urwin R, Zhang Q, Zhou J, Zurth K, Caugant DA, Feavers IM, Achtman M, Spratt BG. Multilocus sequence typing: a portable approach to the identification of clones within populations of pathogenic microorganisms. Proc Natl Acad Sci USA 1998 Mar;95(6):3140-5. DOI PubMed

15. Tsang R, Taha MK. Diagnosis of meningococcal disease. In: Feavers I, Pollard AJ, Sadaranghi M, editors. Handbook of meningococcal disease management. Switzerland: Springer International Publishing; 2016. pp. 45-55. DOI

16. Jolley K. Neisseria sequence typing home page [database]. Wellcome Open Res 2018 (Accessed 2019-01-15);3:124 [version 1; referees: 2 approved]). https://pubmlst.org/ neisseria/ 
17. Tsang RS, Ahmad T, Tyler S, Lefebvre B, Deeks SL, Gilca R, Hoang L, Tyrrell G, Van Caeseele P, Van Domselaar G, Jamieson FB. Whole genome typing of the recently emerged Canadian serogroup W Neisseria meningitidis sequence type 11 clonal complex isolates associated with invasive meningococcal disease. Int J Infect Dis 2018 Apr;69:55-62. DOI PubMed

18. Lahra MM, Enriquez R. Australian Meningococcal Surveillance Programme annual report, 2016. Commun Dis Intell Q Rep 2017 Dec;41(4):E369-82. http://www.health.gov. au/internet/main/publishing.nsf/Content/cdi4104-i PubMed

19. Moore AE, MacNeil JR, Wang X, Joseph SJ, Lorentzson L, Thomas S, Tunali A, Parrott T, Farley MM, Tobin-D'Angelo M. Emergence of localized serogroup $W$ meningococcal disease in the United States - Georgia, 2006-2016. MMWR Morb Mortal Wkly Rep 2018 Aug;67(32):894-7. DOI PubMed

20. Soeters H, Blain A, Chang HY, Whaley M, MacNeil J. Current epidemiology of serogroup $\mathrm{W}$ meningococcal disease United States, 2010-2015. Open Forum Infect Dis 2017;4 Suppl 1:S7. DOI

21. Frank T, Hong E, Mbecko JR, Lombart JP, Taha MK, Rubbo PA. Emergence of Neisseria meningitidis serogroup W, Central African Republic, 2015-2016. Emerg Infect Dis 2018 Nov;24(11):2080-3. DOI PubMed

22. Law DK, Henderson AM, Tsang RS. DNA Sequence analysis of the PorB protein of nonserotypeable serogroup $C$ ET-15 meningococci suggests a potential mutational hot spot on their serotype antigens. J Clin Microbiol 2004 Jun;42(6):2718-23. DOI PubMed

23. Zhou J, Lefebvre B, Deng S, Gilca R, Deceuninck G, Law DK, De Wals P, Tsang RS. Invasive serogroup B Neisseria meningitidis in Quebec, Canada, 2003 to 2010: persistence of the ST-269 clone since it first emerged in 2003. J Clin Microbiol 2012 May;50(5):1545-51. DOI PubMed

24. Jamieson FB, Rawte P, Deeks SL, Zhou J, Law DK, Deng S, Tsang RS. Genetic and antigenic characterization of invasive endemic serogroup B Neisseria meningitidis from Ontario, Canada, in 2001-2010. J Med Microbiol 2013 Jan;62(Pt 1):46-55. DOI PubMed
25. Wang JF, Caugant DA, Morelli G, Koumaré B, Achtman M. Antigenic and epidemiologic properties of the ET-37 complex of Neisseria meningitidis. J Infect Dis 1993 Jun;167(6):1320-9. DOI PubMed

26. Campbell H, Parikh SR, Borrow R, Kaczmarski E, Ramsay ME, Ladhani SN. Presentation with gastrointestinal symptoms and high case fatality associated with group W meningococcal disease (MenW) in teenagers, England, July 2015 to January 2016. Euro Surveill 2016;21(12):30175. DOI PubMed

27. Martin NV, Ong KS, Howden BP, Lahra MM, Lambert SB, Beard FH, Dowse GK, Saul N; Communicable Diseases Network Australia MenW Working Group. Rise in invasive serogroup W meningococcal disease in Australia 2013-2015. Commun Dis Intell Q Rep 2016 Dec;40(4):E454-9.

DOI PubMed

28. Beltrami D, Guilcher P, Longchamp D, Crisinel PA. Meningococcal serogroup W135 epiglottitis in an adolescent patient. BMJ Case Rep 2018 Mar;2018:bcr-2017-223038. DOI PubMed

29. British Columbia Interior Health. Meningococcal outbreak (Okanagan) February 14, 2018 update (Accessed 2019-01-15). https://www.interiorhealth.ca/YourEnvironment/ CommunicableDiseaseControl/Pages/Meningococcal-

Outbreak.aspx

30. BC Gov News. Meningitis protection in B.C. gets a boost. News release. Government of BC: 2019 Apr 25 https://news. gov.bc.ca/releases/2016HLTH0029-000631

31. Agence santé publique du Canada. Programmes de vaccination systématique (et de rattrapage) des provinces et des territoires pour les nourrissons et les enfants au Canada. Ottawa (ON): Gouvernement du Canada. https://www.canada.ca/fr/sante-publique/ services/renseignements-immunisation-provinces-etterritoires/programmes-vaccination-systematique-provin ces-territoires-nourrissons-enfants.html 